\title{
Neuropathies and paraproteins
}

Michael P Lunn MA MBBS FRCP PhD

Centre for Neuromuscular Disease, National Hospital for Neurology and Neurosurgery, Queen Square, London WC1N 3BG

$+442034488121$

michaellunn@nhs.net

\section{Acknowledgements:}

Dr Michael Lunn receives support from the NIHR UCL/UCLH Biomedical Research Centre.

Dr Michael Lunn has no financial conflicts of interest to declare in relation to this paper or its contents.

\section{Keywords:}

Neuropathy, Paraprotein, Neurolymphomatosis, Myelin Associated Glycoprotein 


\section{Key points:}

- The underlying pathogenesis of the haematological origin of the gammopathy causing antiMAG associated paraproteinaemic neuropathy may not be MGUS but more Waldenström's Macroglobulinaemia like opening possibilities for treatment advances.

- Combination treatments of anti-CD20 molecules with other chemotherapy, or new chemotherapeutics such as ibrutinib and ixazomib may transform the treatment of paraprotein associated neuropathies.

- Diagnostic and supportive criteria for POEMS are undergoing re-evaluations and although excellent standards of diagnosis and treatment exist, recent advances in VEGF as a biomarker and imaging findings are of great interest.

- Imaging of nerves in amyloid and neurolymphomatosis continues to develop and advances in PET and MRI modalities are enabling more accurate diagnosis. 


\section{Abstract}

Purpose of the review

There is an increasingly recognised association between haematological and neurological disease. This is especially true in the peripheral nervous system where, to an extent, proof of a link is easier to achieve. The most sensitive low level paraprotein detection methods should always be employed where a paraprotein is suspected. Peripheral nerves can be damaged not only by the immunological targeting of the myelin by the paraprotein, but by deposition (light chain amyloid and cryoglobulins) or direct infiltration (neurolymphomatosis). This has resulted in other defined paraprotein related disease pathogeneses.

Recent findings:

Our opportunities for treating these patients are greater not only through better recognition of disease but also treatments introduced from haematological research. Beyond rituximab, combination therapies, proteasome inhibition and novel biological treatments are being described in haematological practice with early efficacy in neurology. Important developments here should be exploited in neurology to improve outcomes.

\section{Summary:}

This review of the current literature focuses not only on the long term outcome studies in anti-MAG neuropathy, but developments in the diagnosis and treatment of monoclonal gammopathy of undetermined significance and Waldenström's Macroglobulinaemia. 


\section{Introduction:}

Neuropathies as a complication of paraproteinaemias have been recognised for decades. For many years these associations were rare, and attributed to myeloma where serum paraproteins were easily identified by the only available test of agarose gel protein electrophoresis. As techniques for detecting low level paraproteins increased in sensitivity [1], so our understanding of associations as well as pathogenesis increased. In 1981 Kelly described an association of a paraprotein in $10 \%$ of otherwise unexplained neuropathies [2], about four times more than would be expected from the age matched paraprotein prevalence. The class distribution was heavily weighted in favour of IgM paraproteins and once the HNK-1 carbohydrate epitope of myelin associated glycoprotein (MAG) (also found on SGPG/SGLPG and some other peripheral nerve molecules) had been described as a possible target, interest in proving links between neurology and haematology grew rapidly.

There are now a large number of neuropathies with a variety of pathogeneses associated with paraproteins and paraproteinaemic disorders $[3,4]$. These include the IgM associated demyelinating neuropathies associated with monoclonal gammopathy of undetermined significance (MGUS) and Waldenström's Macroglobulinaemia (WM) (which include anti-MAG demyelinating neuropathy, as well as some MMNCB, CANOMAD and a few neuropathies with other defined targets), cryoglobulinaemia and vasculitis, AL-amyloid, POEMS syndrome and neurolymphomatosis. General reviews are already available $[3,4]$ and here I will discuss the most recent evidence about the direct complications of paraproteins; the neuropathies associated with the treatment complications of paraprotein disorders using thalidomide, bortezomib and other chemotherapeutic agents will not be covered.

IgM paraproteinaemic neuropathies. 
Anti-MAG neuropathy remains one of the best defined humorally mediated diseases in neurology

[5]. Many of the modified Witebsky criteria demonstrating pathogenicity of anti-MAG antibodies are fulfilled [6].

The haematology behind the IgM paraprotein in anti-MAG neuropathy has been debated over many years. Anti-MAG antibodies are part of intrinsic repertoire antibody production with sequences possibly matured from germline [7]. Expansion of IgM anti-MAG clones is usually considered part of a monoclonal gammopathy of undetermined significance (MGUS). Mutations in the MYD88 growth regulation gene are associated with Waldenström's Macroglobulinaemia [8]. In patients with IgM anti-MAG neuropathy at least $60 \%$ of patients have the MYD88 mutation detected, and this is likely to be an underestimate [9]. This compares to other IgM MGUS related diseases where MYD88 is seldom found. The implication is that anti-MAG PDPN patients are more WM-like in their pathophysiology and therefore might be investigated and treated in this light.

The International Working Group in Waldenström's Macroglobulinaemia (IWWM) has recently published a consensus statement on the investigation and management of WM related neuropathies [10]. A superficially complex but useful algorithm for diagnosing neuropathies associated with WM summarises the breadth of the neuropathies associated with IgM in WM (Figure 1 - Needs permissions).

The underlying similarity or even commonality with WM is very likely to determine treatment in the near future. Although two randomised controlled trials of rituximab for anti-MAG neuropathy individually failed to show significant benefit $[11,12]$, in meta-analysis rituximab has demonstrable efficacy [13]. Obinutuzumab, a fully humanised anti-CD20 antibody with altered fucosylation to increase NK-cell activation is usually used with chlorambucil for treatment of CLL. Four patients have recently been described as being treated with this agent $[14,15])$. Two of these patients, who were both treatment naïve and treated early in their disease, improved dramatically with concomitant biochemical improvements. The other two had longer disease duration and possibly too much 
axonal loss to improve. Novel anti-CD20 agents such as obinutuzumab in combination with chlorambucil, now seldom used, may be worth revisiting and exploring further.

However other agents beyond, and in addition to, antiCD20 therapy including bendamustine, $1^{\text {st }}, 2^{\text {nd }}$ and $3^{\text {rd }}$ generation proteasome inhibitors (bortezomib, carfilzomib, ixazomib) and the BTK inhibitors (ibrutinib), may all be useful in the future. Ueki et al (2018) and Gomez et al (2016) have recently described beneficial effects of bendamustine combination therapy with rituximab on WM anti-MAG neuropathy $[16,17]$. Neither of the patients treated were typical (one had an associated Bing-Neel syndrome and one was an IgM lambda associated anti-MAG neuropathy whereas the usual association is with an $\operatorname{lgMk})$, but both improved. Bendamustine is relatively toxic and thus proteasome inhibitors aimed at the plasma cell population may be better tolerated. Bortezomib has off target neuropathic effects, but Ixazomib (a $3^{\text {rd }}$ generation proteasome inhibitor) in combination with dexamethasone and rituximab has been shown to be highly effective in the treatment of WM in a phase II study of 26 patients. This study only included 2 neuropathy patients but both improved or stabilised and only mild neuropathic symptoms occurred in others as possible complications [18]. Ixazomib should be investigated for the treatment of neuropathy in the near future. What is required for new trials of treatment is well phenotyped homogeneous cohorts and responsive outcome measures. These are being developed in the IMAGiNe study currently underway [19]. Rituximab has now been used for over 15 years as a single agent in IgM PDPN, and data are emerging on the long term outcomes of cohorts of treated patients [20-23]. Gazzola and colleagues published their centre experience of treating 33 anti-MAG patients with rituximab. 2/3 (18) of these were atypical in their presentation (with subacute or proximal weakness for instance) and these atypical patients were preferentially treated from the whole department cohort. Only $1 / 3$ of the rituximab cohort responded to treatment and the responders tended to be those with proximal weakness and subacute evolution. Gazzola's cohort could not find support for previous findings of response in patients earlier in disease evolution or serum anti-MAG titres or IgM level, but numbers 
were small. Predicting treatment response to target therapy continues to be difficult. Campagnolo et al retrospectively reviewed 33 patients from 5 centres, 2/3 of whom had MGUS and 1/3 WM. Numbers were again small but about half of patients responded, some only to a second dose of rituximab. Response was not correlated to the underlying IgM associated disease, but a lack of response was accompanied by a failure to reduce the overall IgM level after treatment. Some patients in the Campagnolo study required two rituximab treatments, as was found also in the follow up to RiMAG $[12,23]$. lancu-Ferfoglia et al explored the long term (6-year) outcome of a subset of 15 patients in the RiMAG study [12] from a single centre. Over the 6 years since RiMAG, of the seven who had been treated with rituximab in the initial study only two required further treatment, versus six of eight in the placebo treated group. The only clinical difference identifiable between the groups in outcome measures was a significant worsening of the $10 \mathrm{~m}$ walk time in the placebo group. The lack of difference between the groups was attributed more to poorly responsive outcome measures than a lack of actual difference. Overall it seems clear that rituximab is effective in the short and long term in a significant proportion of patients, but that predicting or measuring change remains difficult.

In 2018 the collaborative Francophone Anti-MAG Cohort Group published the largest series of patients in the literature [22]. These patients were defined primarily by the presence of an anti-MAG antibody defined by the BühImann ELISA. Interestingly, possibly as a result of the Bühlmann sensitivity, nearly $16 \%$ did not have demyelinating electrical studies, and a number of patients in this series had other neuropathic diagnoses including Guillain-Barré Syndrome, motor neurone disease and hereditary neuropathy with liability to pressure palsies, all underlining the fact that anti-MAG antibodies occur in the serum of many but do not necessarily result in a neuropathy. There was no link to the Bühlmann titre in terms of disease features or response to treatment. Although there is a broadening of neuropathy phenotypes associated with MAG antibodies, it remains unclear if the antibodies identified by current ELISA technology are pathogenic in all the typical and atypical cases. The potential identification of large numbers of patients without a pathological causative link 
between antibodies and the neuropathy may be partially responsible for the response of only $1 / 3$ of patients to treatment?

Although relatively safe in single use, rituximab is not without potential adverse effects. Svahn et al [22] reported that 11 of 92 rituximab treated patients worsened transiently after treatment, and Doneddu et al [24] also reported a deterioration in tremor. These cases of adverse response join numerous others in the older literature to remind us that increasing effective therapy can potentially be increasingly associated with negative effects.

\section{POEMS}

Until the last 10 years Polyneuropathy, Organomegaly, Endocrinopathy, M-Protein and Skin Changes (POEMS) syndrome was largely neglected as a condition too rare for consideration. Good reviews of POEMS diagnosis and management have recently been published $[25,26]$. The discovery 20 years ago that Vascular Endothelial Growth Factor (VEGF) was strikingly raised in POEMS led to its inclusion as a major diagnostic criterion [25]. Performing a serum VEGF (as well as a serum immunofixation and urine Bence Jones Protein) in all patients with a demyelinating neuropathy might improve POEMS pick up rates, speed diagnosis and reduce disability, by providing an easy biomarker to distinguish POEMS from CIDP. A raised serum VEGF of $>1000 \mathrm{pg} / \mathrm{ml}$, when found in conjunction with an appropriate neuropathy and a lambda light chain paraprotein is $100 \%$ sensitive and $92 \%$ specific to POEMS [27]. However a number of other pathologies can raise VEGF levels including iron deficiency anaemia, bone marrow and bone involving neoplasms and hypoxic disorders and thus these need to be taken into account to prevent overdiagnosis.

Another interesting distinguishing feature of POEMS is that of vascular leak. This is responsible for peripheral oedema, ascites, pleural and alveolar fluid [28] and papilloedema. Fluid accumulation also occurs in more unusual sites, which might possibly be of additional diagnostic help. Ziff et al. confirmed and extended a previous observation that asymptomatic pachymeningeal thickening is 
common in POEMS $[29,30]$. Indeed in a large series of 41 MRI brain scans from 77 POEMS patients, 29 (71\%) had asymptomatic pachymeningeal thickening compared to 0/33 CIDP patients (Figure 2). In four POEMS patients there were significant meningeal collections of fluid. Asymptomatic pachymeningeal thickening may be useful as another supportive minor diagnostic finding assisting differentiation of POEMS from its major differential of CIDP. In addition, this paper also points out that $59 \%$ of POEMS patients has brachial or lumbar plexus thickening and signal change as did $35 \%$ of the CIDP control patients. Thus utilising root and plexus imaging to support a diagnosis of CIDP, as per the EFNS/PNS criteria [31], may well lead to an erroneous CIDP diagnosis and this should be borne in mind.

The treatment of POEMS remains fairly straightforward, albeit confined to centres of excellence. An excellent annual update review was published again in 2019 [25]. Localised disease is treated with radiotherapy, whilst disseminated disease ( $>3$ solitary lesions or bone marrow involvement) is treated with systemic therapy, either melphalan-200 autologous stem cell transplantation (ASCT) or a combination of lenalidomide, melphalan, bortezomib or thalidomide with steroids. The latter two are frequently complicated by worsening neuropathy and thus avoided where possible.

\section{AL Amyloid}

AL Amyloid is a plasma cell proliferative disorder in which light chains deposit in insoluble $\beta$-pleated sheets in many tissues. Its median age of presentation is 62 years and untreated survival is_is_about 24 months. However, the prognosis varies widely from a few months to many years depending upon the severity of cardiac involvement at presentation and for those fit for stem cell transplantation the median survival may exceed 10 years [32]. Light chain (AL) amyloid remains difficult to diagnose especially when there is early and limited organ involvement; early symptoms may be non-specific weight loss and fatigue. Amyloid should always be suspected in 'CIDP' associated with carpal tunnel syndrome, cardiac arrhythmias, nephrotic level proteinuria or notable fatigue [32]. The light chain is lambda in the great majority of cases and biopsy of tissue is required to make the diagnosis. Non- 
invasive diagnosis may be possible with florbetapir or florbetapen-PET scans, more usually used to identify amyloid in Alzheimer's disease. These radiolabel tracers can identify amyloid deposits to target biopsies to lymph nodes or nerve tissue where suspicion is high and a SAP scan is negative $[33,34]$. Diagnosis by targeted biopsy demonstrating typical apple-green birefringent congo red positive deposits is then more likely diagnostic.

Rare localised forms of AL amyloid exist, and if truly local may not affect life expectancy and may not require treatment. When there is systemic AL amyloid and performance status allows, treatment is aimed at destroying the light chain producing clone [35]. Melphalan ASCT is the preferred treatment with best outcomes when patients are carefully selected. Thalidomide and bortezomib are found to be too neurotoxictoxic, and carfilzomib cardiac toxicity limits use. Daratumumab produces haematological responses in $80 \%$ of patients and its further use in neuropathic AL amyloid is awaited.

\section{Cryoglobulinaemic neuropathies}

Another difficult paraproteinaemic neuropathy diagnosis is that of cryoglobulinaemic neuropathy. Cryoglobulins are only found when correct procedures are used to collect and transport blood to the laboratory as some precipitate at only just under $37^{\circ} \mathrm{C}$. Blood should always be collected, transported and pre-processed in the laboratory at $37^{\circ} \mathrm{C}$. Breaks in the warm chain precipitate and discard the cryoglobulin. Cryoglobulins are classified into three types: isolated monoclonal immunoglobulin (Ig) (type I) is associated with B cell lymphoproliferative disorders. Mixed cryoglobulinaemias are composed of either monoclonal and polyclonal Igs (type II) or polyclonal Igs (type III) and are associated with chronic infections (usually hepatitis C virus) and connective tissue disorders [36].

In a 2019 series of 492 cryoglobulinaemias from the Cleveland Clinic, 55\% had a neurological diagnosis, and $27 \%$ of 492 (131) were considered to have a definite or probable cryoglobulinaemia 
link to the neurology. $15 \%$ of these were associated with an MGUS, and $92 \%$ of the 131 had a neuropathy. Interestingly only $13 \%$ were a mononeuritis multiplex phenotype and only $55 \%$ had pain. Most were symmetrical large fibre or small fibre neuropathies and might have been missed without a high level of suspicion of testing for a cryoglobulin [36]. 102 patients all with Type I cryoglobulinaemia were collected in another series [37]. In this paper the additional clues to assist with diagnosis are emphasised, any or all of which may require careful clinical searching. They include the presence of purpura (42\%), and skin ulceration or gangrene (34\%) being common, but also vasomotor symptoms of Raynaud, arthralgias or renal involvement in a significant minority.

The treatment of Type I cryoglobulinaemias follows the principles of many paraproteinaemias related disease, which is to remove or reduce the source. Chemotherapy regimens are directed at the paraproteinaemic disorder and therefore include steroids, alkylating agents, rituximab (often in combinations), novel myeloma therapies or autologous stem cell transplant [37]. To prevent immediate tissue damage, either apheresis or cryofiltration can be tried. Immediate plasmapheresis was effective in 123 of 159 cases in a series from Marson et al.[38]. Cooling the extracorporeal circuit to form a cryogel precipitate may be more effective still [39] but is still yet to be brought into mainstream use.

\section{Neurolymphomatosis}

The direct involvement of peripheral nerves by a paraproteinaemic clone is rare. It is most common with non-Hodgkin's B-Cell lymphomas (NHL), frequently diffuse large B-cell lymphoma (DLBCL) subtypes. Less than $0.5 \%$ of NHL present with neurolymphomatosis [4]. However a painful multiple mononeuropathy in the context of active or previously treated lymphoma, or in the context of a new paraprotein should always prompt suspicion. Histopathological confirmation of the diagnosis is the gold standard, but successfully targeting the right peripheral nerve site requires great experience and some luck. However imaging techniques are developing enabling better localisation. In 2010, the International Primary CNS Lymphoma Working Group identified $77 \%$ of MRI and $84 \%$ of PET-CT were 
abnormal in a series of 50 neurolymphomatosis patients [40]. More recently a single centre collection of 25 patients provided detailed neurography findings supporting the diagnostic utility combined PET-CT and MRI imaging. In proven cases the bone marrow biopsy was negative in 17/19 (89\%) and CSF cytology negative in 16/23 (70\%). Imaging finding were much more helpful and included the presence of a linear or fusiform mass (23/26, 88\%) on 18F-FDG PET/CT which was only moderately avid (average $\mathrm{SuV}_{\text {max: }}$ 7.1 \pm 4.5 , range, 1.5-17.0). On MRI T2-weighted scans masses were hyperintense $(21 / 22,95 \%)$, enhancing $(21 / 22,95 \%)$ and linear or fusiform $(19 / 22,86 \%)$, with associated muscle denervation in a significant number (14/22, 64\%) [41]. Therefore biopsy targeted by imaging may well improve and speed diagnosis in cases where suspicion is high.

\section{Conclusion}

The links between haematologists and neurologists are increasingly frequent as the recognition of paraprotein related disorders affecting peripheral nerves becomes more evident. Many are difficult to diagnose, especially when the pathology is confined to the peripheral nerves alone. However improved biochemical paraprotein detection, and better imaging and targeted biopsy where required will benefit earlier and broader therapeutic opportunities developing in the haematology environment to reduce disability and improve survival. 



\section{References}

[1] Dispenzieri A, Kyle R, Merlini G et al. International Myeloma Working Group guidelines for serumfree light chain analysis in multiple myeloma and related disorders. Leukemia 2009; 23:215-224.

[2] Kelly JJ, Jr, Kyle RA, O'Brien PC, Dyck PJ. The prevalence of monoclonal protein in peripheral neuropathy. Neurology 1981; 31:1480-1483.

[3] Mauermann ML. Neurologic Complications of Lymphoma, Leukemia, and Paraproteinemias. Continuum (Minneap Minn) 2017; 23:669-690.

**[4] Briani C, Visentin A, Campagnolo M et al. Peripheral nervous system involvement in lymphomas. J Peripher Nerv Syst 2019; 24:5-18.

A very good, detailed overview of the neuropathic complications of lymphoma with detailed descriptions of phenotypes.

[5] Nobile-Orazio E, Meucci N, Baldini L et al. Long-term prognosis of neuropathy associated with anti-MAG IgM M-proteins and its relationaship to immune therapies. Brain 2000; 123:710-717.

[6] Witebsky E, Rose NR, Terplan K et al. Journal of the American Medical Association 1957; $164: 1439-1447$.

[7] Spatz LA, Williams M, Brender B et al. DNA sequence analysis and comparison of the variable heavy and light chain regions of two IgM, monoclonal, anti-myelin associated glycoprotein antibodies. Journal of Neuroimmunology 1992; 36:29-39. 
[8] Treon SP, Hunter ZR. A new era for Waldenstrom macroglobulinemia: MYD88 L265P. Blood 2013; 121:4434-4436.

[9] Vos JM, Notermans NC, D'Sa S et al. High prevalence of the MYD88 L265P mutation in IgM antiMAG paraprotein-associated peripheral neuropathy. J Neurol Neurosurg Psychiatry 2018; 89:10071009.

*[10] D'Sa S, Kersten MJ, Castillo JJ et al. Investigation and management of IgM and Waldenstromassociated peripheral neuropathies: recommendations from the IWWM-8 consensus panel. $\mathrm{Br} J$ Haematol 2017; 176:728-742.

An international consensus on the diagnosis and management of neuropathy associated with WM. Contains a decision tree for diagnosis of the commonest associated neuropathic syndromes.

[11] Dalakas MC. B cells as therapeutic targets in autoimmune neurological disorders. Nat.Clin.Pract.Neurol. 2008; 4:557-567.

[12] Leger JM, Viala K, Nicolas G et al. Placebo-controlled trial of rituximab in IgM anti-myelinassociated glycoprotein neuropathy. Neurology 2013; 80:2217-2225.

[13] Lunn MP, Nobile-Orazio E. Immunotherapy for IgM anti-myelin-associated glycoprotein paraprotein-associated peripheral neuropathies. Cochrane Database Syst Rev 2016; 10:CD002827.

[14] Rakocevic G, Martinez-Outschoorn U, Dalakas MC. Obinutuzumab, a potent anti-B-cell agent, for rituximab-unresponsive IgM anti-MAG neuropathy. Neurol Neuroimmunol Neuroinflamm 2018; 5:e460 
[15] Briani C, Visentin A, Salvalaggio A et al. Obinutuzumab, a new anti-CD20 antibody, and chlorambucil are active and effective in anti-myelin-associated glycoprotein antibody polyneuropathy. Eur J Neurol 2019; 26:371-375.

[16] Ueki S, Nakamura M, Sasaki R et al. Beneficial Effect of Bendamustine in a Patient with AntiMAG/SGPG Neuropathy and Bing-Neel Syndrome Associated with Waldenstrom Macroglobulinemia: A Case Report. Case Rep Neurol 2018; 10:88-94.

[17] Gomez A, Hoffman JE. Anti Myelin-Associated-Glycoprotein Antibody Peripheral Neuropathy Response to Combination Chemoimmunotherapy With Bendamustine/Rituximab in a Patient With Biclonal IgM kappa and IgM lambda: Case Report and Review of the Literature. Clin Lymphoma Myeloma Leuk 2016; 16:e101-108.

**[18] Castillo JJ, Meid K, Gustine JN et al. Prospective Clinical Trial of Ixazomib, Dexamethasone, and Rituximab as Primary Therapy in Waldenstrom Macroglobulinemia. Clin Cancer Res 2018; 24:3247-3252.

A crucial paper of a phase II trial of ixazomib, dexamathosone and rituximab in the treatment of Waldenström's Macroglobulinaemia. The trial produced excellent haematological remissions, but only 2 included patients had neuropathy. A further phase III trial is awaited in which hopefully neuropathic outcomes will be collected to identify if there is a therapeutic benefit to this new agent.

[19] Pruppers MHJ, Merkies ISJ, Lunn MPT et al. 230th ENMC International Workshop:: Improving future assessment and research in IgM anti-MAG peripheral neuropathy: A consensus collaborative effort, Naarden, The Netherlands, 24-26 February 2017. Neuromuscul Disord 2017; 27:1065-1072. 
[20] Campagnolo M, Zambello R, Nobile-Orazio E et al. IgM MGUS and Waldenstrom-associated antiMAG neuropathies display similar response to rituximab therapy. J Neurol Neurosurg Psychiatry 2017; 88:1094-1097.

[21] Gazzola S, Delmont E, Franques J et al. Predictive factors of efficacy of rituximab in patients with anti-MAG neuropathy. J Neurol Sci 2017; 377:144-148.

**[22] Svahn J, Petiot P, Antoine JC et al. Anti-MAG antibodies in 202 patients: clinicopathological and therapeutic features. J Neurol Neurosurg Psychiatry 2018; 89:499-505.

The largest cohort of anti-MAG associated patients in the literature collected partly retrospectively from 14 French centres. The report contains detailed data of 202 patients and their response to treatment, found nowhere else in the literature. However it also serves as an example of a disease cohort defined by an antibody, and as the antibody may not always be pathogenic, the clinical features and response to treatment are heterogeneous

[23] lancu Ferfoglia R, Guimaraes-Costa R, Viala K et al. Long-term efficacy of rituximab in IgM antimyelin-associated glycoprotein neuropathy: RIMAG follow-up study. J Peripher Nerv Syst 2016; 21:10-14.

[24] Doneddu PE, Kazmi M, Samuel M et al. Deterioration of tremor after treatment with rituximab in anti-MAG neuropathy. J Neurol Sci 2017; 373:344-345.

**[25] Dispenzieri A. POEMS Syndrome: update on diagnosis, risk-stratification, and management. Am J Hematol 2019.

An excellent summary in the state of the art in POEMS. This paper contains execllent reference material on diagnosis, disease feaatures, trials, biomarkers and disease outcomes. 
[26] Keddie S, Lunn MP. POEMS syndrome. Curr Opin Neurol 2018; 31:551-558.

*[27] Pihan M, Keddie S, D'Sa S et al. Raised VEGF: High sensitivity and specificity in the diagnosis of POEMS syndrome. Neurol Neuroimmunol Neuroinflamm 2018; 5:e486.

All diagnostic tests and biomarkers can be misused. This paper illustrates the utility of VEGF in the diagnosis of POEMS considering all the appropriate differential diagnoses as well as the confounders for finding a raised VEGF level. VEGF should be utilised with a search for an appropriate monoclonal plasma cell disorder and interpreted in the light of that and the neuropathy as in the published criteria.

[28] Al-Mayoof O, Al Sughaiyer H, Abuomar W, Khan M. POEMS syndrome: a rare cause of exudative ascites and chronic peripheral neuropathy. BMJ Case Rep 2017; 2017.

[29] Briani C, Fedrigo M, Manara R et al. Pachymeningeal involvement in POEMS syndrome: MRI and histopathological study. J Neurol Neurosurg Psychiatry 2012; 83:33-37.

*[30] Ziff OJ, Hoskote C, Keddie S et al. Frequent central nervous system, pachymeningeal and plexus MRI changes in POEMS syndrome. J Neurol 2019; 266:1067-1072.

It would not be usual to image the brain in a peripheral nerve disorder where there are no clinical indicators to do so. However vascular leak occurs in the meninges as it does in other tissues and in some respects is easier to identify there than at some other sites. Furthermore the presence of signal change and enhancement in the plexi in POEMS, currently suggested as a diagnostic feature of CIDP means that the current criteria could actively result in POEMS misdiagnosis as CIDP. This paper has important implications for future POEMS and CIDP criteria sets. 
[31] Van den Bergh PY, Hadden RD, Bouche P et al. European Federation of Neurological Societies/Peripheral Nerve Society guideline on management of chronic inflammatory demyelinating polyradiculoneuropathy: report of a joint task force of the European Federation of Neurological Societies and the Peripheral Nerve Society - first revision. Eur J Neurol 2010; 17:356-363.

[32] Gertz MA. Immunoglobulin light chain amyloidosis diagnosis and treatment algorithm 2018. Blood Cancer J 2018; 8:44.

*[33] Broski SM, Spinner RJ, Howe BM et al. 18F-Florbetapir and 18F-FDG PET/CT in Systemic Immunoglobulin Light Chain Amyloidosis Involving the Peripheral Nerves. Clin Nucl Med 2016; 41:e115-117.

Novel imaging methods may result in an otherwise cryptic diagnosis being made and treatment being offered much earlier. This repurposing of an amyloid PET tracer can be very effective.

[34] Fox TA, Lunn M, Wechalekar A et al. [(18)F]Florbetaben PET-CT confirms AL amyloidosis in a patient with Waldenstrom's Macroglobulinemia. Haematologica 2018; 103:e322-e324.

[35] Vaxman I, Bernstine $\mathrm{H}$, Kleinstern $\mathrm{G}$ et al. FDG PET/CT as a diagnostic and prognostic tool for the evaluation of marginal zone lymphoma. Hematol Oncol 2019; 37:168-175.

**[36] Feldman L, Dhamne M, Li Y. Neurologic manifestations associated with cryoglobulinemia: A single center experience. J Neurol Sci 2019; 398:121-127.

An impressively large series of patients with cryoglobulinaemia diligently collected over many years. A quarter of the patients have definite or probable neurology associated with the cryoglobulin illustrating the significant pathogenic potential of precipitating protein. Most of the neurology is unsurprisingly neuropathic, but given the pathology is vasculitic the lack of pain in most and the 
majority being symmetrical sensorimotor neuropathy make this an important illustration of the need to keep a broad open mind in considering diagnoses.

[37] Sidana S, Rajkumar SV, Dispenzieri A et al. Clinical presentation and outcomes of patients with type 1 monoclonal cryoglobulinemia. Am J Hematol 2017; 92:668-673.

[38] Marson P, Monti G, Montani F et al. Apheresis treatment of cryoglobulinemic vasculitis: A multicentre cohort study of 159 patients. Transfus Apher Sci 2018; 57:639-645.

[39] Taniyama Y, Nakatani Y, Matsuoka T et al. Efficacy of Cryofiltration for Treatment of Mixed Cryoglobulinemia: A Report of Four Cases. Ther Apher Dial 2017; 21:238-242.

[40] Grisariu S, Avni B, Batchelor TT et al. Neurolymphomatosis: an International Primary CNS Lymphoma Collaborative Group report. Blood 2010; 115:5005-5011.

**[41] DeVries AH, Howe BM, Spinner RJ, Broski SM. B-cell peripheral neurolymphomatosis: MRI and (18)F-FDG PET/CT imaging characteristics. Skeletal Radiol 2019.

The utility of an imaging modality is only as good as its resolution powers. Using both PET-CT and MRI together, targeted to suspicious areas increases diagnostic yield and leads to a refinement of diagnosis. In combination with more aggressive chemotherapy regimens that allow better intraneural disease control earlier diagnosis could lead to better outcomes. 

Figure 2: a Coronal T2w image showing apparent widening of subdural planes over bifrontal convexities. b Coronal post-gadolinium T1w equivalent plane image from the same patient demonstrating thick enhancing dura in this area. c Post-gadolinium T1w coronal image showing two opposing layers of enhancing dura. The central non-enhancing area is effusion: the "double falx" appearance. $\mathbf{d}$ Postgadolinium T1w image demonstrating diffuse enhancement of intracranial pachymeninges. e Post-gadolinium T1w image demonstrating focal enhancement of intracranial pachymeninges. $\mathbf{f}$ Coronal FLAIR showing moderate degree of small vessel disease. $\mathbf{g}$ Postgadolinium T1w image of the cervical spine level C5 showing intradural leptomeningeal enhancement of cervical nerve roots. $\mathbf{h}$ Thickened and enhancing roots/proximal lumbar plexus on post-gadolinium T1w coronal image 


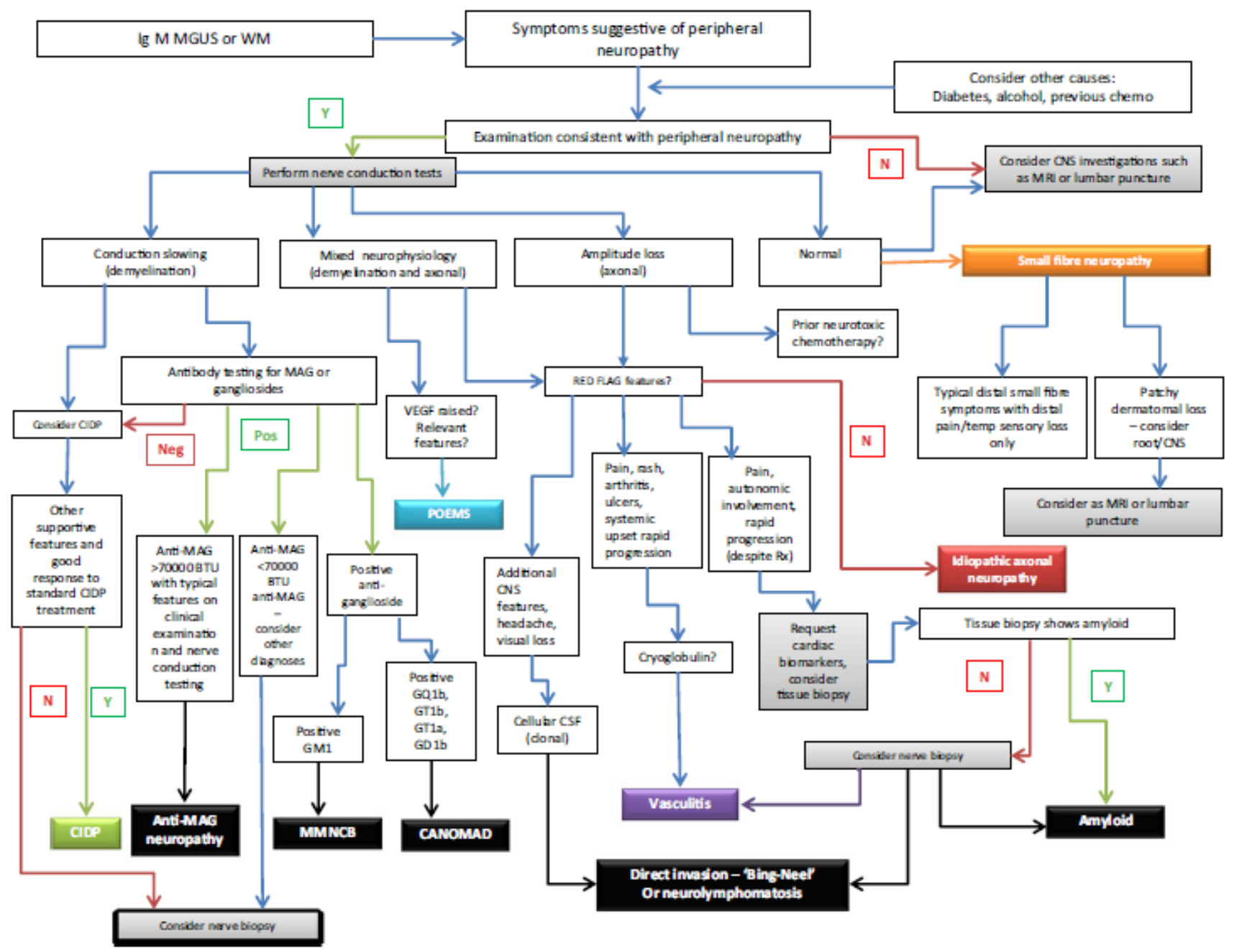

Fig 1. Schematic decision tree for the evaluation of IgM-associated peripheral neuropathies. Boxes in black denote IgM-related diagnoses. Other colours denote relevant differential diagnoses. BTU, Bühlmann units; CANOMAD, chronic ataxic neuropathy with ophthalmoplegia, M-protein, cold agglutinins and disialosyl ganglioside antibodies; CIDP, chronic inflammatory demyelinating polyneuropathy; CNS, central nervous system; CSF, cerebrospinal fluid; IgM, immunoglobulin M; MAG, myelin associated glycoprotein; MGUS, monoclonal gammopathy of undetermined significance; MMNCB, multifocal motor neuropathy with conduction block; MRI, magnetic resonance imaging; POEMS, polyneuropathy, organomegaly, endocrinopathy, monoclonal plasma cell disorder, skin changes;Rx, radiotherapy; WM, waldenström macroglobulinaemia. 


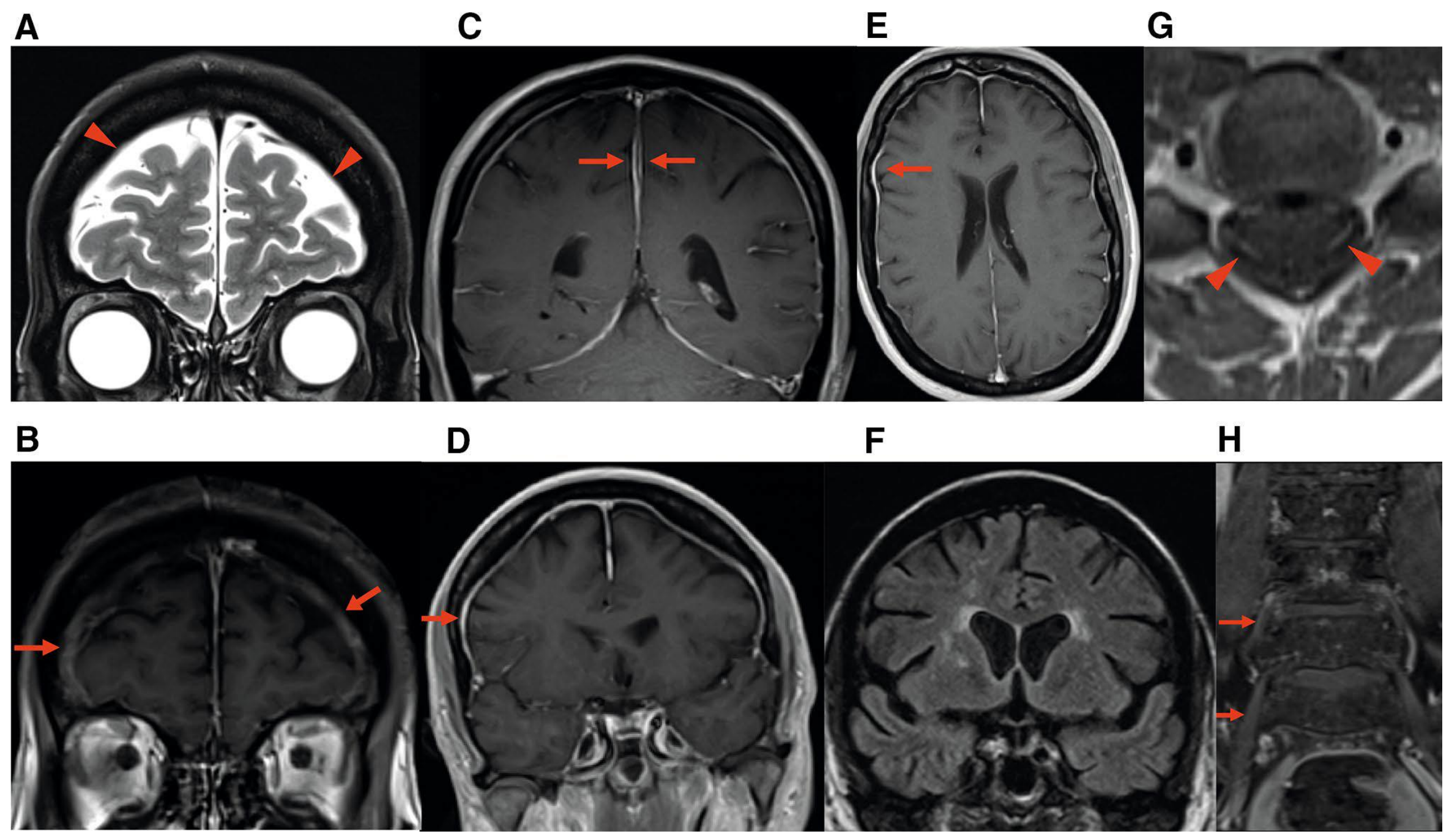

